\title{
The Path Planning Algorithm Of Triangle Grid Scan For Localization In Wireless Sensor Network
}

\author{
Yuehchen Lin ${ }^{1, a}$ and Jingsin Liu ${ }^{1, b}$ \\ ${ }^{1}$ Institute of Information Science, Academia Sinica, Taipei, Taiwan \\ ahyde@iis.sinica.edu.tw, ${ }^{b}$ liu@iis.sinica.edu.tw
}

Keywords: Localizability, Trilateration, Localization, Mobile Robot, Wireless Sensor Networks.

\begin{abstract}
Localization is an important process in wireless sensor networks (WSNs). In this paper, we present a localization algorithm based on triangle grid scan (TGS) method. The objective of this research is to provide an affordable mean for localization using wireless area network Wi-Fi technology with mobile robot. Experimental study reveals that the proposed TGS mechanism effectively guides the mobile anchor to move along an efficient path.
\end{abstract}

\section{Introduction}

Developing a good deployment and path planning algorithm is one of the most important issues for an efficient WSN. In the literature, we propose a localization algorithm with a mobile anchor node based on trilateration in WSNs. Existing sensor node can be classified into three categories: stationary sensor, mobie sensor, and mobile robot. Trilateration method uses distance from nearby AP with known Media Access Control (MAC) addresses, calculated from signal strength values, to approximate the distance to the user. In most cases, Sensor Network only needs to know the distance between each other to operate relative applications in network. As a result of this, if we have an algorithm to calculate the relative distance of sensors, we can apply it to sensor network in order to avoid interference of uncertainties and achieve practical value. This is also the reason why the position algorithm without sensors of known nodal coordinates gradually wins its own spotlight. Our TGS method is compared with SCAN, LMAT[1], and HILBERT[2] algorithms. Simulation results show that the performance of our TGS algorithm is better than that of other similar algorithms. Another deployment alternative $[3,4]$ is to use the robot to deploy static sensors. The robot explores the environment and deploys a stationary sensor to the target location from time to time. The robot deployment can achieve full coverage with fewer sensors.

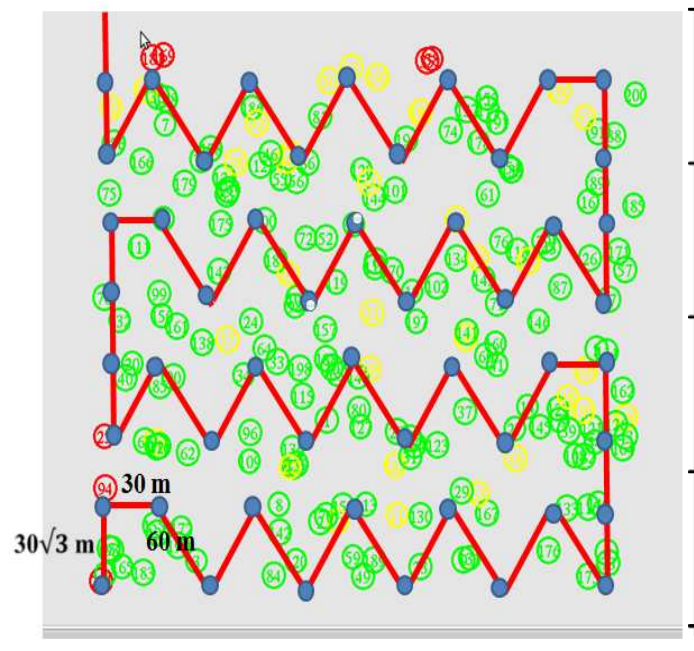

(a)

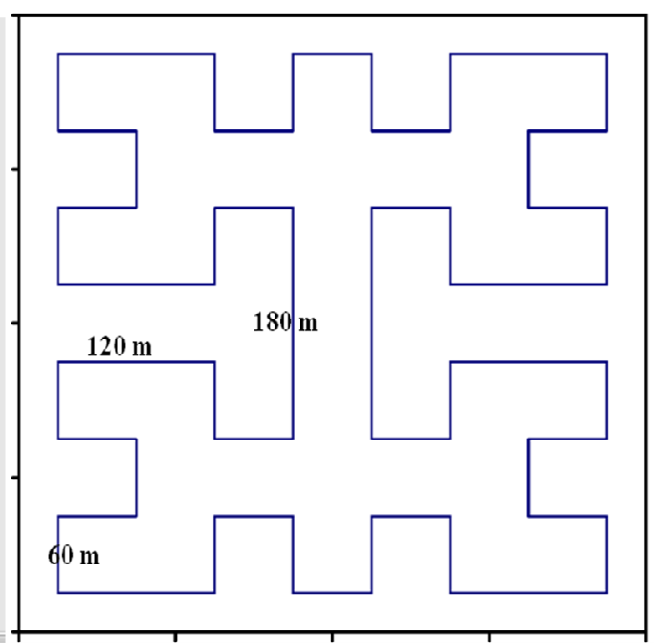

(b)

Fig. 1 (a)LMAT and (b) HILBERT method for path planning process 


\section{Triangle Grid Scan Algorithm}

Environment. The WSN environment has the size $\mathrm{W}^{*} \mathrm{H}$. All sensor nodes and the mobile anchor node have the same communication radius $r$. In [3] ,the author test the square anchor placement and triangle anchor placement, and they find that positioning of sensors in a WSN can be done by observing the properties of a triangle. we adopt the triangle grid to design the path which mobile anchor move along, as shown in Fig. 2.

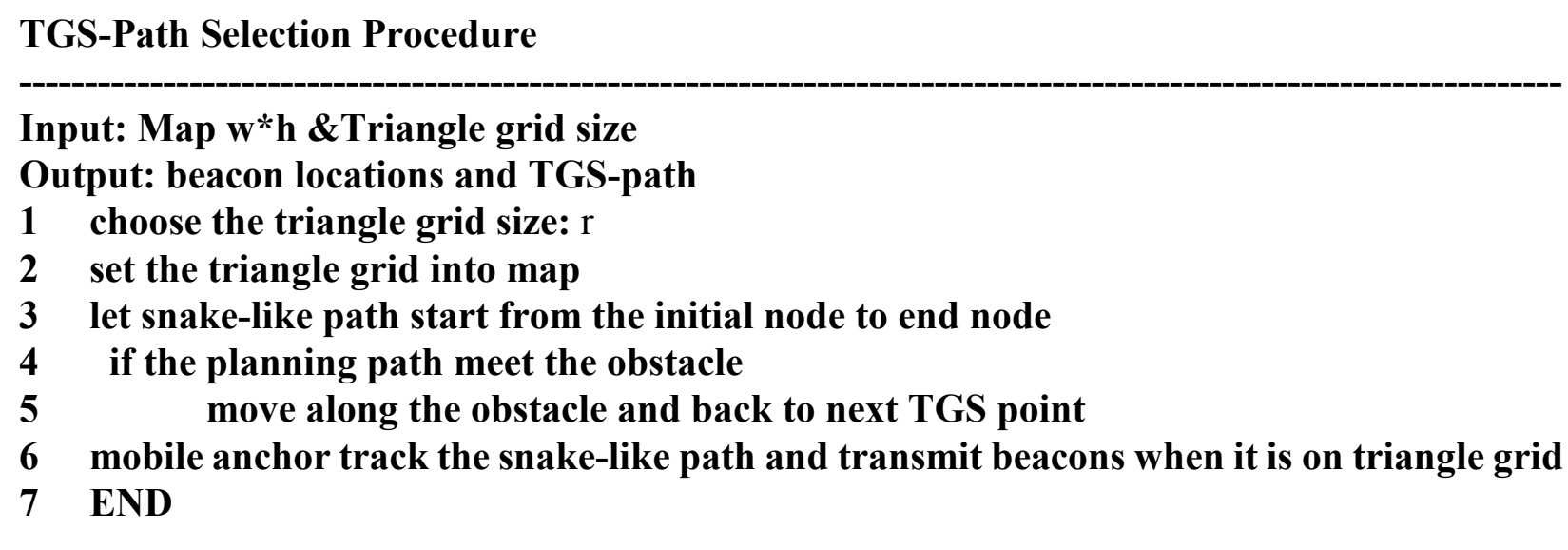

Triangle Grid For Trilatertion. In our algorithm, the entire network is divided into many non-overlapping triangle cells (Fig. 2). In each cell a group of N, nodes is deployed. Usually nodes in each cell reside according to some probability distribution function (PDF). Here we assume in each cell nodes are uniformly deployed. A trilateration system also consists of a series of joined or overlapping triangles. However, for trilateration the lengths of all the sides of the triangle are measured and few directions or angles are measured to establish azimuth. Trilateration has become feasible with the development of electronic distance measuring (EDM) equipment which has made possible the measurement of all lengths with high order of accuracy under almost all field conditions. The basic principle of trilateration is that the position of an object can be uniquely determined bymeasuring the distances to three reference positions. Trilatertion-based approaches, however, recognize only asubset (called trilateration extension) of globally rigid graphs. As a compromise, trilateration is proposed for testing localizability based on the fact that the location of an object can be determined if the distances to three references are known. Accordingly, it is possible to identify localizable nodes in a network by iteratively applying trilaterations.

Analysis. The maps has the size width $(\mathrm{W})^{*}$ height $(\mathrm{H})$ and the radio range of sensors is $\mathrm{R}$, so we can decompose it into two parts. The lenth of TGS in width can be :

$[\mathrm{W} /(\sqrt{3} / 2 * \mathrm{R}) \times \mathrm{R}] \times(\mathrm{H} / \mathrm{R}-1)+1 / 2 *(\sqrt{3} * \mathrm{R}) \times \mathrm{W} /(\sqrt{3} * \mathrm{R})$.

In the another hand, The lenth of TGS in height can be:

$(\mathrm{H} / \mathrm{R}) \mathrm{xR}$ or $(\mathrm{H} / \mathrm{R}-2)+2 \Delta \mathrm{L}$

,where $\Delta \mathrm{L}$ is defined by[1].

Coverage. Coverage reflects how well a sensor network observesthe physical space; thus, it can be viewed as the quality of service of the sensing function. Previous designs fall into two categories. The probabilistic approaches analyze the node density for ensuring appropriate coverage statistically, but essentially have no guarantee on the result. In contrast, the geometric approaches are able to obtain accurate and reliable results, in which locations are essential. TGS method provide a good graph coverage for mobile anchor in WSN. 


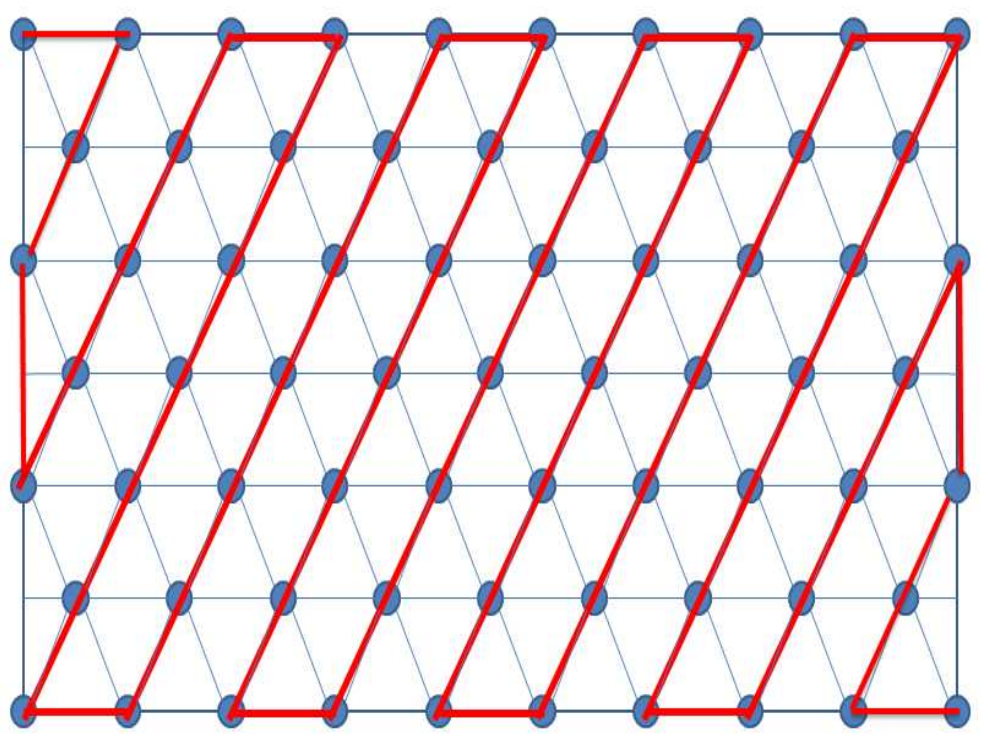

Fig. 2 TGS method of design process
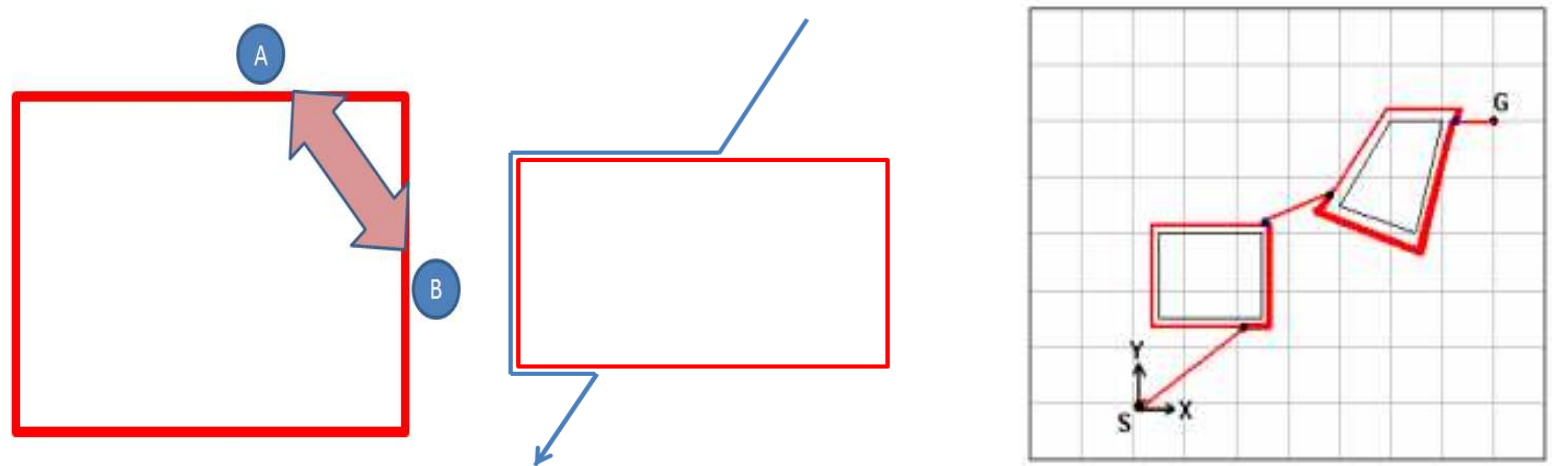

Fig. 3 The design process of encountering obstacle method.

Obstacle Solution. When the robot encounters obstacles, it moves around them to deploy sensors and eliminate the impact of obstacles on deployment. During the execution of the deployment task, the robot switches between steady and obstacle states, depending on whether it comes across obstacles, as shown in Fig. 3.

\section{System Model And Experiments}

- In simulation analysis, we use ns2 simulator. We test our algorithm using the simulation tool and then compare the result to that of SCAN, HILBERT, and LMAT, see Fig. 4 below. A number of 200 sensors are to be deployed in the square area. The travelling speed of an anchor node is $2 \mathrm{~m} / \mathrm{s}(72 \mathrm{~km} / \mathrm{hr})$.

- The computations were carried out on a server with a $2.66 \mathrm{GHz}$ eight processor and 16 GB RAM. 


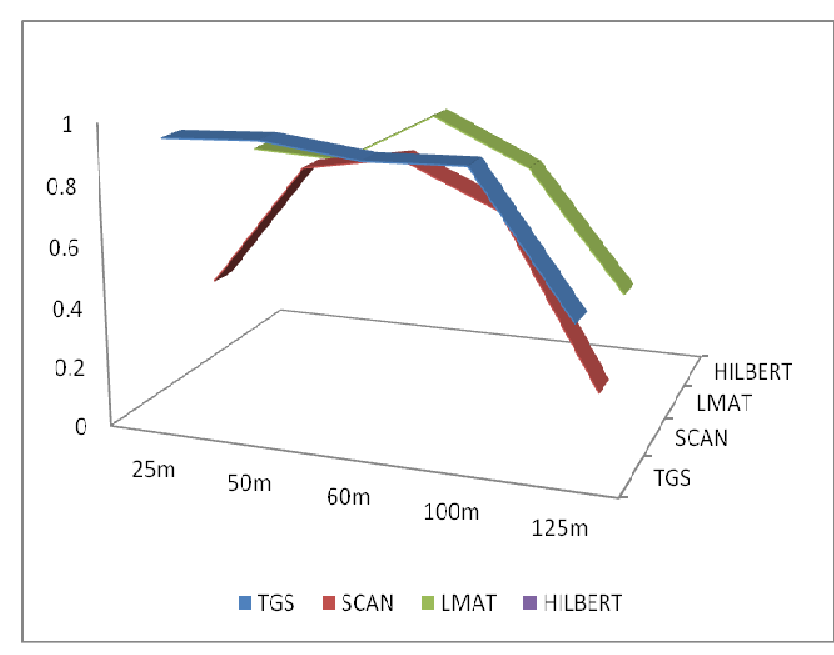

(a)

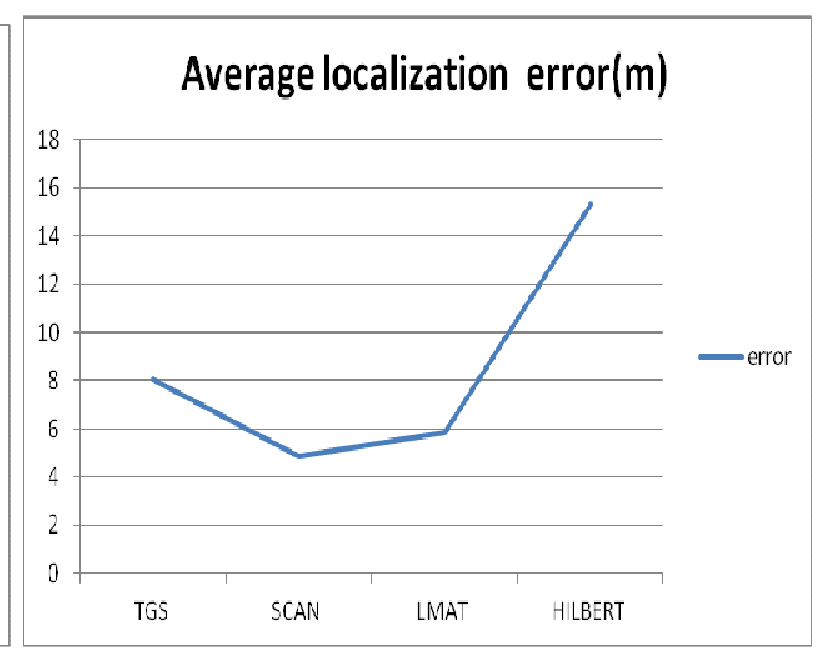

(b)

Fig. 4 (a)Comparative the percentage of localizable nodes and (b) Localization error

In the scenario, a number of 200 sensors are deployed in a area conforming to a random distribution. Moreover, the map size is $500 * 433 \mathrm{~m}^{2}$. As shown in Fig. 4(a),we test different radio range for four methods. In Fig. 4(a), We run several simulation experiments using different sensor radio range that ranges from $25 \mathrm{~m}$ to $125 \mathrm{~m}$. The row means radio range which is multiple of 25 meter. It is obvious that the percentage of localizable nodes of TGS is better than other methods, and the percentage of localizable nodes by TGS is $95 \%$. TGS outperforms other by recognizing a larger number of localizable nodes. About localization error, Fig. 4(b) show the average localization error, and the average localization error by TGS is $7.49 \mathrm{~m}$. We also find that the obstacle in map don't affect the the percentage of localizable nodes in TGS method much, because there is no sensor nodes in the obstacle area.

\section{Conclusions}

In this thesis study, we propose a mobile routing algorithm with scanning in the triangle grid architecture. During the initializing phase of the sensor network, we introduce path planning and scheduling mechanism. The location information of mobile sink node is received by the TGS method. Furthermore, the effect of square and triangle anchor placement on the algorithm performance is examined, and triangle anchor placement is shown to have better performance with fewer anchor requirements.

\section{References}

[1] G. Han, H. Xu and J. Jiang: Wirel. Commun. Mob. Comput.(2011)

[2] D. Koutsonikolas, S.M. Das, Y.C. Hu : Computer Communications 30 (2007) ,p.2577-2592

[3] Y.Wang, X. Wang in: IEEE TRANSACTIONS ON PARALLEL AND DISTRIBUTED SYSTEMS, VOL. 20, NO. 10, (2009)

[4] C.Y. Chang, J.P. Sheu, Y.C. Chen, and S.W. Chang :IEEE TRANSACTIONS ON SYSTEMS, MAN, AND CYBERNETICS—PART A: SYSTEMS AND HUMANS, VOL. 39, NO. 4, (2009). 